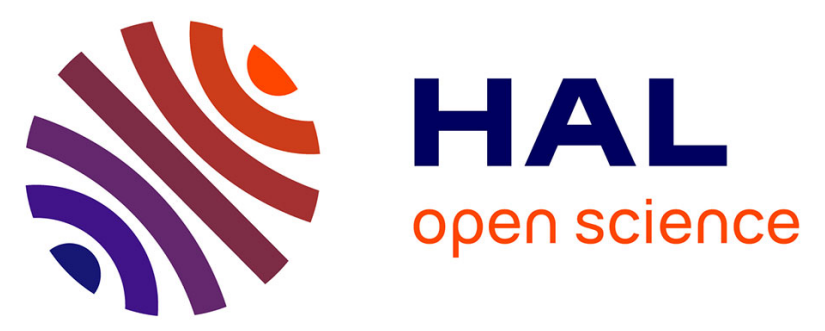

\title{
Persistent FDG/PET CT uptake in idiopathic retroperitoneal fibrosis helps identifying patients at a higher risk for relapse
}

Gabriel Morin, Arthur Mageau, Khadija Benali, Remi Bertinchamp, Eve Piekarski, Quentin Raimbourg, Jean-Francois Alexandra, Tiphaine Goulenok, Damien van Gysel, Thomas Papo, et al.

\section{To cite this version:}

Gabriel Morin, Arthur Mageau, Khadija Benali, Remi Bertinchamp, Eve Piekarski, et al.. Persistent FDG/PET CT uptake in idiopathic retroperitoneal fibrosis helps identifying patients at a higher risk for relapse. European Journal of Internal Medicine, 2019, 62, pp.67 - 71. 10.1016/j.ejim.2019.01.019 . hal-03486176

\section{HAL Id: hal-03486176 https://hal.science/hal-03486176}

Submitted on 20 Dec 2021

HAL is a multi-disciplinary open access archive for the deposit and dissemination of scientific research documents, whether they are published or not. The documents may come from teaching and research institutions in France or abroad, or from public or private research centers.
L'archive ouverte pluridisciplinaire HAL, est destinée au dépôt et à la diffusion de documents scientifiques de niveau recherche, publiés ou non, émanant des établissements d'enseignement et de recherche français ou étrangers, des laboratoires publics ou privés.

\section{(ㄷ)(1) $\$$}

Distributed under a Creative Commons Attribution - NonCommerciall 4.0 International 


\section{Persistent FDG/PET CT uptake in idiopathic retroperitoneal fibrosis helps identifying} patients at a higher risk for relapse.

Gabriel Morin ${ }^{\mathrm{a}}$, Arthur Mageau ${ }^{\mathrm{a}}$, Khadija Benali ${ }^{\mathrm{b}}$, Remi Bertinchamp ${ }^{\mathrm{a}}$, Eve Piekarski ${ }^{\mathrm{b}}$, Quentin Raimbourg ${ }^{\mathrm{c}}$, Jean-Francois Alexandra ${ }^{\mathrm{a}}$, Tiphaine Goulenok ${ }^{\mathrm{a}}$, Damien van Gysel ${ }^{\mathrm{d}}$, Thomas Papo ${ }^{\mathrm{a}, \mathrm{e}, \mathrm{f}}$, Karim Sacre ${ }^{\mathrm{a}, \mathrm{e}, \mathrm{f}}$

a-Département de Médecine Interne, Hôpital Bichat, Université Paris Diderot, PRES Sorbonne Paris Cité, Assistance Publique Hôpitaux de Paris, Paris, France

b-Département de Médecine Nucléaire, Hôpital Bichat, Université Paris Diderot, PRES Sorbonne Paris Cité, Assistance Publique Hôpitaux de Paris, Paris, France

c-Département de Nephrologie, Hôpital Bichat, Université Paris Diderot, PRES Sorbonne Paris Cité, Assistance Publique Hôpitaux de Paris, Paris, France

d-Département d'Information Médicale, Hôpital Bichat, Université Paris Diderot, PRES Sorbonne Paris Cité, Assistance Publique Hôpitaux de Paris, Paris, France

e-Département Hospitalo-Universitaire FIRE (Fibrosis, Inflammation and Remodelling in

Renal and Respiratory Diseases), Paris, France

f-INSERM U1149, Paris, France

\section{Corresponding author:}

Professor Karim Sacre, MD, PhD

Department of Internal Medicine, Bichat Hospital, APHP

46 rue Henri Huchard, 75018, Paris, France

Phone : 33140256019 Fax : 33140258845 karim.sacre@aphp.fr

The authors declare no conflicts of interest.

This work was supported by the Département Hospitalo-Universitaire DHU "FIRE 


\section{ABSTRACT}

Background: The aim of this study was to evaluate the prognostic value of persistent retroperitoneal fibrosis FDG uptake using FDG/PET CT in patients with idiopathic retroperitoneal fibrosis (IRF).

Methods In this monocentric retrospective cohort study, all patients admitted for IRF from January 2009 to December 2017 underwent a FDG/PET CT at diagnosis and during follow up. Metabolic activity of IRF was assessed by retroperitoneal fibrosis FDG uptake measured as maximal standardized uptake value (SUVmax). The primary outcome was IRF relapse rate during follow-up.

Results: 23 consecutive patients (54.7 [36.9-89] years, 73.9\% of men) diagnosed with IRF had FDG/PET CT imaging performed at diagnosis, 3.1 [1-8.7] months (i.e $1^{\text {st }}$ evaluation) and 10.4 [4.9-17.5] months (i.e $2^{\text {nd }}$ evaluation) after diagnosis. High FDG retroperitoneal fibrosis uptake was present in all patients at diagnosis (SUVmax 6.5 [3.8-11.9]) and persisted in 16 (69.6\%; SUVmax 3.65 [2.1-5.4]) and 12 (52.2\%; SUVmax 3.75 [2.7-7.8]) patients, at $1^{\text {st }}$ and $2^{\text {nd }}$ evaluation respectively. All but one patient had received steroids at IRF diagnosis and 21 $(91.3 \%)$ were in complete remission at both $1^{\text {st }}$ and $2^{\text {nd }}$ evaluation. During a median follow-up period of 38.7 [3-106.9] months, 6 (26.1\%) patients suffered IRF relapse that occurred 15.7 [9.2-42.8] months after diagnosis. Multivariate analysis showed that only persistent retroperitoneal fibrosis FDG uptake at 2nd evaluation was associated with IRF relapse $(\mathrm{p}=0.046)$.

Conclusions In IRF, persistent retroperitoneal fibrosis FDG uptake during follow up is associated with clinical outcome. FDG/PET CT may help to better stratify the risk of relapse and target therapy in IRF.

KEYWORD idiopathic retroperitoneal fibrosis; FDG/PET CT; relapse 


\section{INTRODUCTION}

Idiopathic retroperitoneal fibrosis (IRF) is a rare inflammatory disorder, characterized by the presence of a fibro-inflammatory tissue surrounding abdominal vessels and often obstructing the ureters ${ }^{1-3}$. Treatment of IRF is based on steroids that are highly effective to obtain

remission ${ }^{4}$. However, the high rate of relapse once steroids are withdrawn led to prolonged therapy in most patients ${ }^{4}$. Being able to select patients according to their risk of relapse would probably help physicians to optimize maintenance treatment.

18F-fluorodeoxyglucose-positron emission tomography (FDG/PET CT) is a useful tool for detection of inflammatory changes in numerous inflammatory diseases including IRF ${ }^{5-10}$. To our best knowledge, no study tested FDG-PET/CT in order to predict outcome in IRF.

We hypothesized that long-standing FDG/PET CT uptake of retroperitoneal fibrosis might be a predictor of relapse. The aim of this study was to analyze both retroperitoneal fibrosis FDG uptake at diagnosis and follow up along with clinical outcome in patients admitted for IRF.

\section{PATIENTS and METHODS}

\section{Patients}

All charts of consecutive adult patients hospitalized over an 8-year period (from January 2009 to December 2017) for a new diagnosis of IRF in the Department of Internal Medicine (Bichat Hospital, Paris, France) were retrospectively reviewed. Exclusion criteria were previous treatment for retroperitoneal fibrosis and secondary retroperitoneal fibrosis including drugrelated retroperitoneal fibrosis, tuberculosis, neoplasia and Erdheim-Chester disease. IRF was diagnosed by abdominal CT or MRI, following commonly accepted radiological criteria ${ }^{1,2,11}$. Retroperitoneal biopsy was done in patients with suspected secondary forms or atypical mass localization. All patients with IRF underwent FDG/PET CT at diagnosis and during follow up. IRF relapse was defined by the presence of recurrent or new-onset disease related 
symptoms (abdominal/back pain, fatigue, weight loss and/or low-grade fever), hydronephrosis and/or mass enlargement on CT or MRI associated with an increase in CRP values. IRF complete remission was defined by the disappearance of initial symptoms associated with normal CRP, decreased/stabilization of serum creatinine level and decreased/stabilization of the retroperitoneal mass on CT or MRI. The primary outcome measure was IRF relapse rate over follow up.

\section{Ethical statement}

Our study is a human non-interventional retrospective study where 1-subjects were not assigned to treatment, 2-subjects were assigned to a diagnosis strategy within current practice, 3-epidemiological methods were used to analyze the data, and 4- information used in the study were collected for clinical care. According to the Public Health French Law (art L 1121-1-1, art L 1121-1-2), written consent are not required for human non-interventional studies. For ethical consideration, patients were however informed that data that was collected in medical records might be used for research study in accordance to privacy rule. The study protocol conforms to the ethical guidelines of the 1975 Declaration of Helsinki and was approved by the local ethics committee (Institutional Review Board IRB 00006477 of HUPNVS, Paris 7 University, AP-HP).

\section{Data collection}

International Classification of Disease codes (ICD-10) for retroperitoneal fibrosis (N135, C786, M728) were used for patients screening. Data were extracted from the French Diagnosis Related Groups (DRG) based information system (PMSI, programme de médicalisation des systèmes d'information) databases. Information regarding eligibility condition were obtained from medical records. All patients underwent blood sampling for 
markers of inflammation (CRP and white blood cell count), abdominal CT or MRI and FDG/PET CT at diagnosis and during follow up. Demographic data, medical history, laboratory findings, imaging findings and follow up data were retrieved from medical records.

\section{Treatment protocol}

According to clinical practice, first line therapy for IRF was steroid alone (prednisone) given orally at a starting dose of $1 \mathrm{mg} / \mathrm{kg} /$ day for 4 weeks. Prednisone dose was then tapered by $10 \mathrm{mg}$ every 4 weeks until dose was $20 \mathrm{mg} /$ day, then by $5 \mathrm{mg}$ every 4 weeks until $10 \mathrm{mg} /$ day and eventually by $1 \mathrm{mg}$ every 4 weeks until $7 \mathrm{mg} /$ day. Depending of IRF remission status and steroids-related toxicity over follow up, low dose steroids ( $7 \mathrm{mg} /$ day) was prolonged 12 to 36 months. Treatment of relapse consisted of immunosuppressive drugs (IS), mainly methotrexate (15 to $20 \mathrm{mg} / \mathrm{week}$ ) or rituximab ( $1 \mathrm{~g}$ as an infusion on days 0 and 14 ), in association with steroids.

\section{FDG/PET CT retroperitoneal fibrosis uptake measurement}

FDG/PET CT was performed using a hybrid PET CT scanner (Discovery 690, GE Healthcare) after an overnight fast. Images were acquired from the skull base to the proximal thighs ( 3 min per bed position), 60 min after intravenous administration of $3.5 \mathrm{MBq} / \mathrm{kg} 18 \mathrm{~F}$ FDG. In diabetic patients, glucose should be $<12.0 \mathrm{mmol} / \mathrm{L}$ at the time of 18 -FDG injection. Low-dose CT (100 keV and $140 \mathrm{~mA}$ with current modulation system) without contrast enhancement was acquired for anatomic correlation and attenuation correction of the PET data. PET images were reconstructed using 3-dimensional time-of-flight ordered-subsets expectation maximization with and without attenuation correction and reoriented in axial, sagittal, and coronal slices ( $3 \mathrm{~mm}$ cross-section thickness and 256x256 matrix for a visual field of view of $60 \mathrm{~cm}$ ). Reconstructed images were displayed on an ADW workstation (GE 
Healthcare) for visual analysis. PET (attenuation corrected and non-corrected) images alone and co-registrated PET CT images were analyzed by two senior nuclear medicine physicians to detect foci of non-physiological hypermetabolism. The presence of a retroperitoneal fibrosis FDG uptake was defined as a circumferential linear FDG uptake matching with the circumferential soft-tissue thickening around the distal aorta and/or common iliac vessels on CT. The 18-FDG uptake was measured by generating a volume of interest to calculate the maximal standardized uptake value. Only the SUVmax has been considered in the results to avoid partial volume effect.

\section{Statistical analysis}

Data are expressed as median with range for variable with non-Gaussian distribution or frequency (percentage) as appropriate. Comparisons between patients with and without IRF relapse were made using Mann-Whitney tests for continuous variables and $\mathrm{Chi}^{2}$ tests for categorical variables. For the multivariate analysis, a logistic regression procedure including all variables that had a significant bivariate relation (as defined by a $\mathrm{p}$ value of less than 0.05 ) with the primary outcome was performed. Kaplan-Meïer method was used to represent IRF relapse according to the FDG retroperitoneal fibrosis uptake (i.e. with or without FDG uptake) observed at $2^{\text {nd }}$ evaluation. Two-sided $\mathrm{P}$ values of less than 0.05 were considered to indicate statistical significance. Statistical analyses were performed with R software version 3.1.0.

\section{RESULTS}

\section{Patient characteristics}

From January 2009 to December 2017, 31 consecutive patients admitted for retroperitoneal fibrosis were eligible for the study. Eight patients were excluded because retroperitoneal fibrosis was already known and previously treated $(n=7)$ or secondary to endometrial 
neoplasia $(n=1)$. Overall, 23 patients with a new diagnosis of IRF were studied. Seventeen patients (73.9\%) were male and the median age was 54.7 [34.4-89] years. At IRF diagnosis, $20(86.9 \%)$ and 13 (56.5\%) had abdominal/back pain and systemic symptoms (fatigue, weight loss and/or low-grade fever), respectively. CRP was high (>20mg/L) in $12(52.2 \%)$ patients. High serum creatinine concentration $(>100 \mu \mathrm{mol} / \mathrm{L})$ caused by ureteral obstruction requiring JJ stent at diagnosis was observed in $10(43.5 \%)$ patients. Retroperitoneal fibrosis was periaortoiliac, peri-aortic and peri-iliac in $11(47.8 \%), 10(43.5 \%)$ and $2(8.7 \%)$ cases, respectively with a median fibrosis thickness of 16 [10-57] $\mathrm{mm}$ on imaging diagnostic. In 8 (34.8\%) cases, retroperitoneal fibrosis was surrounding an aneurysmal abdominal aorta. Retroperitoneal biopsy was performed in only 10 (43.5\%) patients and revealed IgG4-related disease in 3 cases (Table 1).

\section{FDG/PET CT retroperitoneal fibrosis uptake}

FDG/PET CT imaging was performed at diagnosis, $3.1[1-8.7]$ months $\left(1^{\text {st }}\right.$ evaluation) then 10.4 [4.9-17.5] months ( $2^{\text {nd }}$ evaluation) after IRF diagnosis in all patients. Reproducibility (proportion of agreement of $100 \%$ ) between the readers to assess the metabolic activity of retroperitoneal fibrosis was excellent at each evaluation. At diagnosis, retroperitoneal fibrosis FDG uptake was abnormal in $23(100 \%)$ patients with a median maximum standardized uptake value (SUVmax) of 6.5 [3.8-11.9]. All but one patient received steroids (prednisone, initial dose $1 \mathrm{mg} / \mathrm{kg}$ daily) that was started after the FDG/PET CT imaging performed at diagnosis in all cases. All but 2 patients $(91.3 \%)$ were in complete remission at the $1^{\text {st }}$ evaluation and $2^{\text {nd }}$ imaging evaluation, while FDG retroperitoneal fibrosis uptake was still high in 16 (69.6\%; SUVmax 3.65 [2.1-5.4]) and 12 (52.2\%; SUVmax 3.75 [2.7-7.8]) patients, respectively (Figure 1 and Table 2). Interestingly, metabolic activity of retroperitoneal fibrosis 
assessed by retroperitoneal fibrosis FDG uptake SUVmax significantly correlated with CRP level measured at the concomitant time ( $\mathrm{p}=0.0004, \mathrm{R}^{2}=0.26$; Figure 2$)$.

\section{FDG/PET CT retroperitoneal fibrosis uptake and clinical outcome}

During a median follow-up of 38.7 [10-106.9] months, 6 (26.1\%) patients experienced IRF relapse. In all cases, relapse occurred after the $2^{\text {nd }}$ FDG/PET CT imaging evaluation. The median time to relapse from diagnosis and $2^{\text {nd }}$ evaluation was 15.7 [9.2-42.8] and 2.7 [0.828.8] months, respectively. At relapse, all 6 patients were still receiving prednisone at a median dose of $8.5[5-15] \mathrm{mg} /$ day.

Univariate analysis showed that CRP level at diagnosis $(\mathrm{p}=0.025)$, lack of complete remission at $1^{\text {st }}$ and $2^{\text {nd }}$ evaluation $(\mathrm{p}=0.059)$ and persistent retroperitoneal fibrosis FDG uptake at 2nd evaluation $(p=0.014)$ were associated with IRF relapse (Tables 3$)$. In the multivariate analysis, only persistent retroperitoneal fibrosis FDG uptake at $2^{\text {nd }}$ evaluation $(p=0.046)$ was associated with IRF relapse (Table 3). Sensitivity, specificity, negative predictive value and positive predictive value of persistent retroperitoneal fibrosis FDG uptake at $2^{\text {nd }}$ evaluation to predict IRF relapse were respectively $100 \%, 60 \%, 100 \%$ and 50\%. In Kaplan-Meier analysis,

retroperitoneal fibrosis FDG uptake at $2^{\text {nd }}$ evaluation was significantly associated with IRF relapse ( $\mathrm{p}=0.017$; HR 7.1 95\%CI [1.4-35.9]) (Figure 3).

Treatment of relapse consisted of increased prednisone daily dose in all cases associated immunosuppressive drugs (IS) in 4 patients, including methotrexate $(n=2)$ and rituximab $(\mathrm{n}=2)$. At last visit, all patients were in complete remission while having received steroids for 26.9 [7.6-106.9] months. Thirteen (56.5\%) patients were still treated by steroids. In $3(13 \%)$ patients, steroids were associated with IS. Two (16.7\%) patients died from severe infection. 


\section{DISCUSSION}

IRF has a chronic relapsing course. To define optimal duration of treatment would need to better identify patients at high risk of relapse. In this study, we show that persistent retroperitoneal fibrosis FDG uptake on FDG/PET CT is an independent factor associated with IRF relapse.

Our findings provide relevant insights for clinical practice. First, relapse rate is high (around $25 \%$ of patients), consistent with previous report ${ }^{4}$. Second, we confirm that retroperitoneal fibrosis FDG uptake at diagnosis and during follow up correlates with C-reactive protein levels, and is thus indicative of disease activity ${ }^{6,8}$. Third, remission -defined by the disappearance of initial symptoms along with the decrease/stabilization in fibrosis size, renal function improvement and the normalization of inflammatory parameters- is reached in most patients receiving steroids as expected ${ }^{4,6}$ but does not preclude smoldering inflammatory activity. Fourth, FDG/PET CT is confirmed as highly sensitive to detect residual disease activity in patients in apparent remission ${ }^{7,8}$. Last, no relapse was observed among patients who had no residual retroperitoneal fibrosis FDG uptake during follow up. This finding suggests that duration of treatment might be shortened in patients in whom initial retroperitoneal fibrosis FDG uptake disappears under steroids.

Our study has several limitations. Its retrospective scheme from a single center and the small sample size limit the statistical power and impede the ability to generalize the findings. Because IRF is rare and proper randomized trials are difficult to mount, most studies in the field are retrospective with a limited number of patients. On the other hand, our series is relatively large with IRF features that fit with the results obtained in other studies ${ }^{1-4,8,12-15}$. Also, since the method using standardized uptake values (SUV) to assess IRF metabolic activity is quantitative, it may allow comparisons across different studies. Although histological examination of the retroperitoneal tissue appears as the gold standard IRF 
diagnosis tool, only $10(43.5 \%)$ patients had retroperitoneal biopsy at diagnosis. In our study, we believe that typical imaging findings ${ }^{16}$, clear-cut response to steroids and protracted follow-up did rule out secondary forms of retroperitoneal fibrosis ${ }^{6}$. Retroperitoneal fibrosis belongs to the IgG4-related disease spectrum ${ }^{17}$. Retroperitoneal biopsy showed intense IgG4+ plasma cell infiltration consistent with IgG4-related disease in only 3 cases. Of note, none of the 20 remaining "non-biopsy" patients had typical extra-retroperitoneal IgG4-related features. Moreover, no striking difference has been observed between IgG4-related and IgG4negative retroperitoneal fibrosis in clinical, imaging or outcome published data.

In conclusion, persistent FDG/PET CT uptake is associated with IRF outcome and may predict the risk of relapse. Furthers studies are needed to assess whether such tool may help to better stratify treatment and allow early steroid discontinuation according to relapse risk identified by FDG/PET CT.

\section{ACKNOWLEDGEMENTS}

We are thankful to Drs M.P. Chauveheid and A. Dossier for their help with patients' screening. This work was supported by the Département Hospitalo-Universitaire DHU "FIRE".

\section{CONFLICT OF INTEREST}

The authors declare no conflicts of interest. Results presented in this paper have not been published previously in whole or part

\section{AUTHORS' CONTRIBUTIONS}

KS had full access to all of the data in the study and takes responsibility for the integrity of the data and the accuracy of the data analysis. Study design: KS. Acquisition of data: GM, 
AM, KB, RB, EP, QR, JFA, TG, DG, TP, KS. Analysis and interpretation of data: GM, AM, KB, RB, EP, QR, JFA, TG, DG, TP, KS. Manuscript preparation: GM, AM, TP, KS. Manuscript revision: GM, AM, KB, RB, EP, QR, JFA, TG, DG, TP, KS.

\section{FUNDING STATEMENT}

None

\section{REFERENCES}

1. van Bommel EF, Jansen I, Hendriksz TR, Aarnoudse AL. Idiopathic retroperitoneal fibrosis: prospective evaluation of incidence and clinicoradiologic presentation. Medicine (Baltimore) 2009, 88:193-201.

2. Corradi D, Maestri R, Palmisano A, Bosio S, Greco P, Manenti L, et al. Idiopathic retroperitoneal fibrosis: clinicopathologic features and differential diagnosis. Kidney Int 2007, 72:742-753.

3. Lugosi M, Sacre K, Lidove O, Chauveheid MP, Brihaye B, Laissy JP, et al. Long-term follow-up of a French cohort of retroperitoneal fibrosis. Rev Med Interne 2013, 34:591-599.

4. Vaglio A, Palmisano A, Alberici F, Maggiore U, Ferretti S, Cobelli R, et al. Prednisone versus tamoxifen in patients with idiopathic retroperitoneal fibrosis: an open-label randomised controlled trial. Lancet 2011, 378:338-346.

5. Caiafa RO, Vinuesa AS, Izquierdo RS, Brufau BP, Ayuso Colella JR, Molina CN. Retroperitoneal fibrosis: role of imaging in diagnosis and follow-up. Radiographics 2013, $33: 535-552$.

6. Piccoli GB, Consiglio V, Arena V, Pelosi E, Anastasios D, Ragni F, et al. Positron emission tomography as a tool for the 'tailored' management of retroperitoneal fibrosis: a nephro-urological experience. Nephrol Dial Transplant 2010, 25:2603-2610. 
7. Moroni G, Castellani M, Balzani A, Dore R, Bonelli N, Longhi S, et al. The value of (18)FFDG PET/CT in the assessment of active idiopathic retroperitoneal fibrosis. Eur J Nucl Med Mol Imaging 2012, 39:1635-1642.

8. Jansen I, Hendriksz TR, Han SH, Huiskes AW, van Bommel EF. (18)F-fluorodeoxyglucose position emission tomography (FDG-PET) for monitoring disease activity and treatment response in idiopathic retroperitoneal fibrosis. Eur J Intern Med 2010, 21:216-221.

9. Lariviere D, Benali K, Coustet B, Pasi N, Hyafil F, Klein I, et al. Positron emission tomography and computed tomography angiography for the diagnosis of giant cell arteritis: A real-life prospective study. Medicine (Baltimore) 2016, 95:e4146.

10. Gerardin C, Mageau A, Benali K, Jouan F, Ducrocq G, Alexandra JF, et al. Increased FDG-PET/CT pericardial uptake identifies acute pericarditis patients at high risk for relapse. Int J Cardiol 2018, 271:192-194.

11. Scheel PJ, Jr., Feeley N. Retroperitoneal fibrosis: the clinical, laboratory, and radiographic presentation. Medicine (Baltimore) 2009, 88:202-207.

12. Salvarani C, Pipitone N, Versari A, Vaglio A, Serafini D, Bajocchi G, et al. Positron emission tomography (PET): evaluation of chronic periaortitis. Arthritis Rheum 2005, 53:298-303.

13. Ha YJ, Jung SJ, Lee KH, Lee SW, Lee SK, Park YB. Retroperitoneal fibrosis in 27 Korean patients: single center experience. J Korean Med Sci 2011, 26:985-990.

14. Fernandez-Codina A, Martinez-Valle F, Castro-Marrero J, Detorres I, Vilardell-Tarres M, Ordi-Ros J. Idiopathic retroperitoneal fibrosis: a clinicopathological study in 24 Spanish cases. Clin Rheumatol 2013, 32:889-893.

15. Accorsi Buttini E, Maritati F1, Vaglio A. $\left[{ }^{18} \mathrm{~F}\right]$-Fluorodeoxyglucose Positron Emission Tomography and Response to Therapy in Idiopathic Retroperitoneal Fibrosis. Eur Urol. 2018;73:145-146 
16. Arrive L, Hricak H, Tavares NJ, Miller TR. Malignant versus nonmalignant retroperitoneal fibrosis: differentiation with MR imaging. Radiology 1989, 172:139-143.

17. Stone JH, Zen Y, Deshpande V. IgG4-related disease. N Engl J Med 2012, 366:539-551. 


\section{TABLES}

Table 1 Characteristics of patients at baseline

\begin{tabular}{l|ccc} 
& Relapse & No Relapse & \\
& $(\mathrm{n}=6)$ & $(\mathrm{n}=17)$ & $\mathrm{P}$ \\
\hline Age, years & $53.3[41.2-61.3]$ & $57.1[34.4-89]$ & 0.575 \\
Men, $\mathrm{n}(\%)$ & $4(66.7)$ & $13(76.5)$ & 0.638 \\
Clinical manifestation at diagnosis, $\mathrm{n}(\%)$ & & & \\
Abdominal/back pain, $\mathrm{n}(\%)$ & $6(100)$ & $14(82.4)$ & 0.539 \\
Systemic symptoms, $\mathrm{n}(\%)$ & $4(66.7)$ & $9(52.9)$ & 0.560 \\
Laboratory findings at diagnosis & & & \\
CRP level, mg/L & $40[10-221]$ & $17[3-40]$ & 0.025 \\
High CRP level at diagnosis, $\mathrm{n}(\%)$ & $5(83.3)$ & $7(41.2)$ & 0.076 \\
Serum creatinin level, umol/L & $143.5[90-2254]$ & $79[54-560]$ & 0.441 \\
High creatinin level at diagnosis, $\mathrm{n}(\%)$ & $3(50)$ & $7(41.2)$ & 0.708 \\
Imaging findings at diagnosis & & & \\
Aneurysmal abdominal aorta, $\mathrm{n}(\%)$ & $3(50)$ & $5(29.4)$ & 0.170 \\
Periaortoiliac RPF, $\mathrm{n}(\%)$ & $3(50)$ & $8(47)$ & 0.725 \\
Isolated periaortic, $\mathrm{n}(\%)$ & $3(50)$ & $7(41.2)$ & 0.708 \\
Isolated peri-iliac, $\mathrm{n}(\%)$ & $0(0)$ & $2(11.8)$ & 1 \\
Maximal RPF thickness, mm & $16[15-57]$ & $14.5[10-35]$ & 0.465 \\
FDG/PET RPF uptake, $\mathrm{n}(\%)$ & $6(100)$ & $17(100)$ & 1 \\
SUVmax & $10.1[5.5-11.9]$ & $5.4[3.2-10.7]$ & 0.280 \\
Retroperitoneal biopsy at diagnosis, $\mathrm{n}(\%)$ & $3(50)$ & $7(41.2)$ & 0.708 \\
IgG4, $\mathrm{n}(\%)$ & $1(16.7)$ & $2(11.8)$ & 0.759 \\
Treatment at diagnosis & & & \\
Steroids, $\mathrm{n}(\%)$ & $6(100)$ & $16(94.1)$ & 1 \\
JJ stents, $\mathrm{n}(\%)$ & $3(50)$ & $7(41.2)$ & 0.708
\end{tabular}

Values are $\mathrm{n}(\%)$ or median (range); Systemic symptoms referred to fatigue, weight loss and/or low-grade fever; High CRP (C-reactive protein) referred to CRP>20mg/L; High serum creatinine concentration referred to creatinine $>100 \mu \mathrm{mol} / \mathrm{L}$; RPF, retroperitoneal fibrosis; FDG/PET, fluorodeoxyglucose-positron emission tomography; SUVmax, maximal standardized uptake value; Complete remission defined by the disappearance of initial symptoms associated with normal CRP, decreased/stabilization of serum creatinine level and decreased/stabilization of the retroperitoneal mass on CT or MRI 
Table 2 Clinical outcome and FDG/PET CT retroperitoneal fibrosis uptake during

follow up

\begin{tabular}{l|ccc} 
& Relapse & No Relapse \\
$(\mathrm{n}=6)$ & $(\mathrm{n}=17)$ & $\mathrm{P}$ \\
\hline First evaluation & $3[1.1-8.7]$ & $3.1[1.2-12.9]$ & 0.418 \\
$\quad$ Delay form diagnosis, months & $4(66.7)$ & $17(100)$ & 0.059 \\
Complete remission, $\mathrm{n}(\%)$ & $6(100)$ & $10(58.8)$ & 0.124 \\
FDG/PET RPF uptake, $\mathrm{n}(\%)$ & $3.4[3.1-3.7]$ & $4.1[2.1-5.4]$ & 0.779 \\
SUVmax & & & \\
Second evaluation & $11.1[8.9-17.3]$ & $10.4[2.8-60.3]$ & 0.018 \\
Delay form diagnosis, months & $4(66.7)$ & $17(100)$ & 0.059 \\
Complete remission, $\mathrm{n}(\%)$ & $6(100)$ & $6(35.3)$ & 0.014 \\
FDG/PET RPF uptake, n (\%) & $5.3[2.7-7.8]$ & $3.4[3.2-4.5]$ & 0.084 \\
SUVmax & & & \\
Last evaluation/Follow up & $47.9[26.9-106.9]$ & $33.8[10-71.5]$ & 0.764 \\
Delay from diagnosis, months & $6(100)$ & $17(100)$ & 1 \\
Complete remission, $\mathrm{n}(\%)$ & $45.5[26.9-106.9]$ & $23.4[7.6-56.6]$ & 0.810 \\
Cumulative months of steroids & $2(33.3)$ & $8(47)$ & 0.484 \\
Steroids withdrawn, $\mathrm{n}(\%)$ & $4(66.7)$ & 0 & 0.002 \\
Immunosuppressive drugs at any time, $\mathrm{n}(\%)$ & 0 & $2(11.8)$ & 1
\end{tabular}

FDG/PET, fluorodeoxyglucose-positron emission tomography; RPF, retroperitoneal fibrosis;

SUVmax, maximal standardized uptake value; Complete remission defined by the disappearance of initial symptoms associated with normal CRP, decreased/stabilization of serum creatinine level and decreased/stabilization of the retroperitoneal mass on CT or MRI 
Table 3 Univariate and multivariate analysis of risk factors for IRF relapse

\begin{tabular}{l|ccc|ccc} 
& \multicolumn{3}{|c|}{ Univariate analysis } & \multicolumn{3}{c}{ Multivariate analysis * } \\
& Estimated OR & IC 95 & P & Estimated OR & IC 95 & p \\
\hline FDG uptake at 2nd evaluation $^{* *}$ & 19 & $1,73-\mathrm{X}$ & 0.0016 & 12.41 & $1.037-\mathrm{X}$ & 0.046 \\
Complete remission at 2nd evaluation $^{* *}$ & 0.051 & $0,003-0,77$ & 0.031 & 0.37 & $0.002-11.10$ & 0.56 \\
Delay from diagnosis to 2nd evaluation $^{* * *}$ & 1.01 & $0,90-1,07$ & 0.99 & $\mathrm{NC}$ & $\mathrm{NC}$ & $\mathrm{NC}$ \\
High CRP at diagnosis $^{*}$ & 5.13 & $0,78-58,2$ & 0.089 & $\mathrm{NC}$ & $\mathrm{NC}$ & $\mathrm{NC}$ \\
CRP at diagnosis & 1.02 & $1,00-1,24$ & 0.048 & 1,01 & $0,97-1,19$ & 0.67
\end{tabular}

* logistic regression using FDG uptake at 2nd evaluation, complete remission at 2nd evaluation, and CRP at diagnosis

**2nd evaluation performed 10.4 [4.9-17.5] months after IRF diagnosis

*** delay from the diagnosis to 2nd evaluation using FDG/PET CT imaging

$\mathrm{X}$ referred to value $>100$ 


\section{LEGENDS TO FIGURES}

Figure 1 Retroperitoneal fibrosis FDG uptake at diagnosis and during follow up

Maximum Intensity Projection (MIP) (A1), axial PET (A2), axial fused PET-CT (A3), sagittal PET(A4) and fused sagittal PET-CT (A5) images at diagnosis. Maximum Intensity Projection (MIP) (B1), axial PET (B2), axial fused PET-CT (B3), sagittal PET (B4) and fused sagittal PET-CT (B5) images after 3 months of steroids ( $1^{\text {st }}$ evaluation). Abnormal tracer uptakes involving the retroperitoneal fibrosis are shown (red/green arrows). Maximum standard uptake value (SUVmax) was 10.4 and 3.5 at diagnosis and at $1^{\text {st }}$ evaluation, respectively

\section{Figure 2 SUVmax and serum CRP level in patients with IRF}

Metabolic activity of retroperitoneal fibrosis measured by SUVmax correlated with serum CRP level measured at the concomitant time $\left(\mathrm{R}^{2}=0.26 ; \mathrm{p}=0.0004\right)$.

\section{Figure 3 Kaplan-Meier Curves of Study Population}

Patients with persistent FDG retroperitoneal fibrosis uptake on FDG/PET imaging performed 10.4 [4.9-17.5] months after IRF diagnosis ( $2^{\text {nd }}$ evaluation) (FDG/PET+) had higher rates of IRF relapse than patients without FDG-PET retroperitoneal fibrosis uptake (FDG/PET-); $\mathrm{p}=0.017 ;$ HR 7.1 95\%CI [1.4-35.9] 

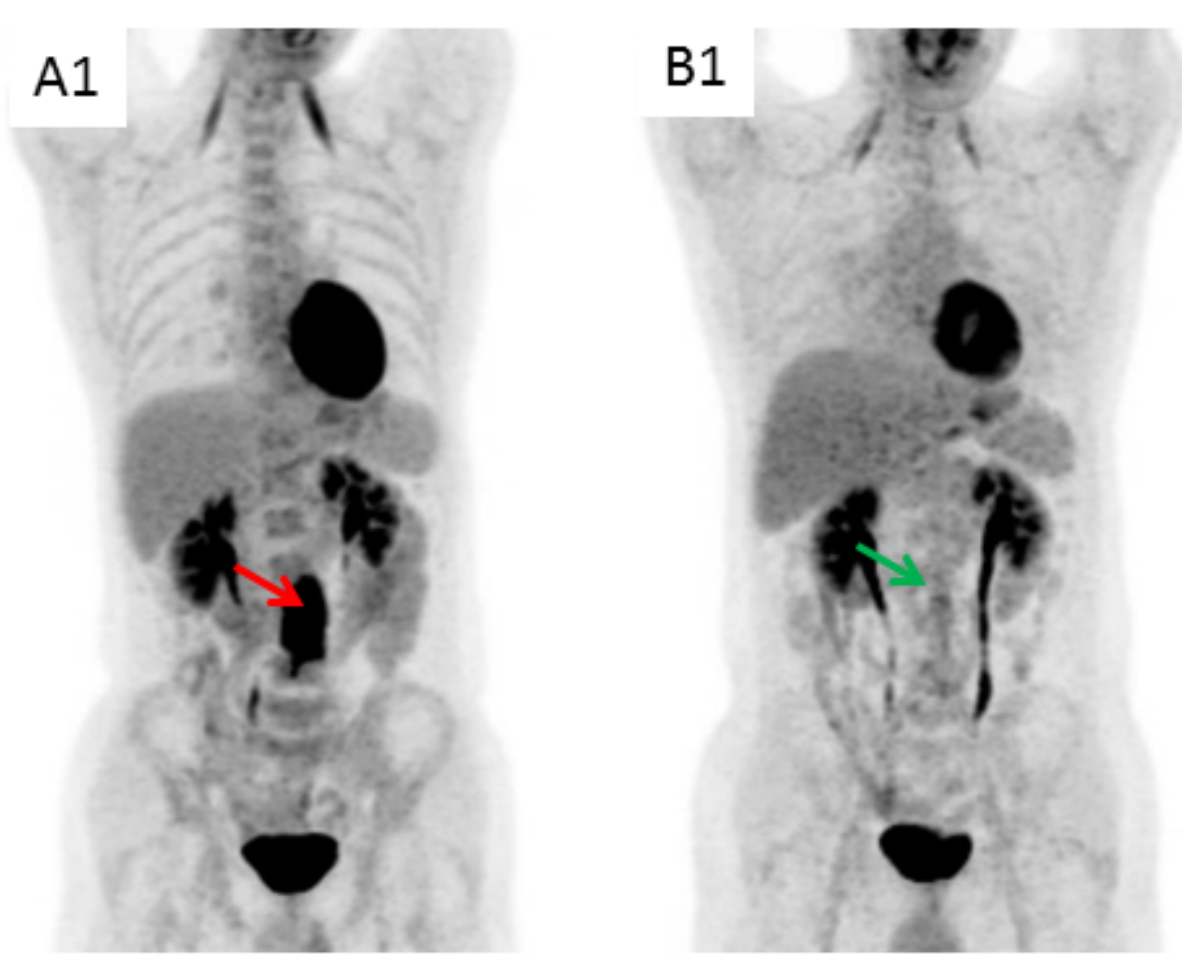

A2

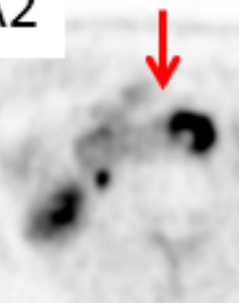

B2

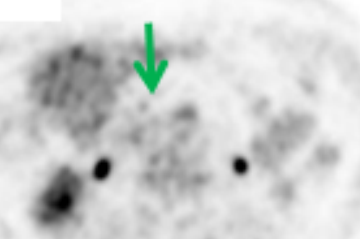

A4
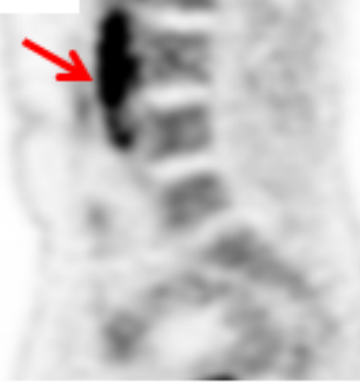

B4

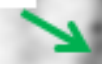

A3

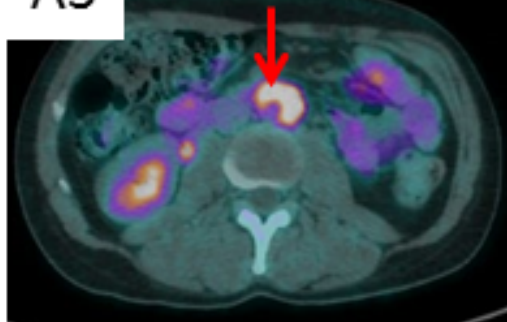

\section{B3}

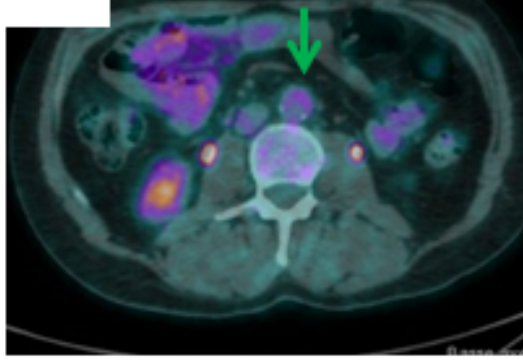

$$
\text { A5 }
$$
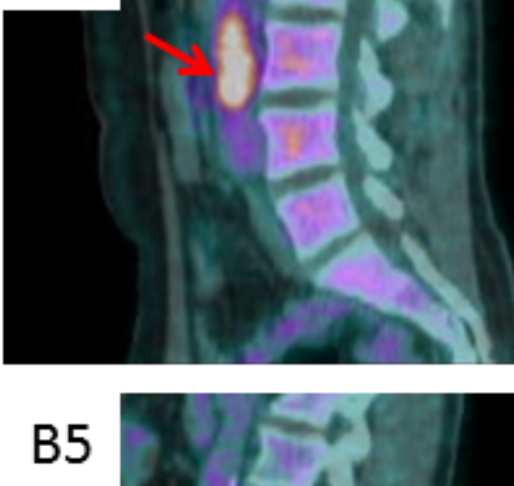


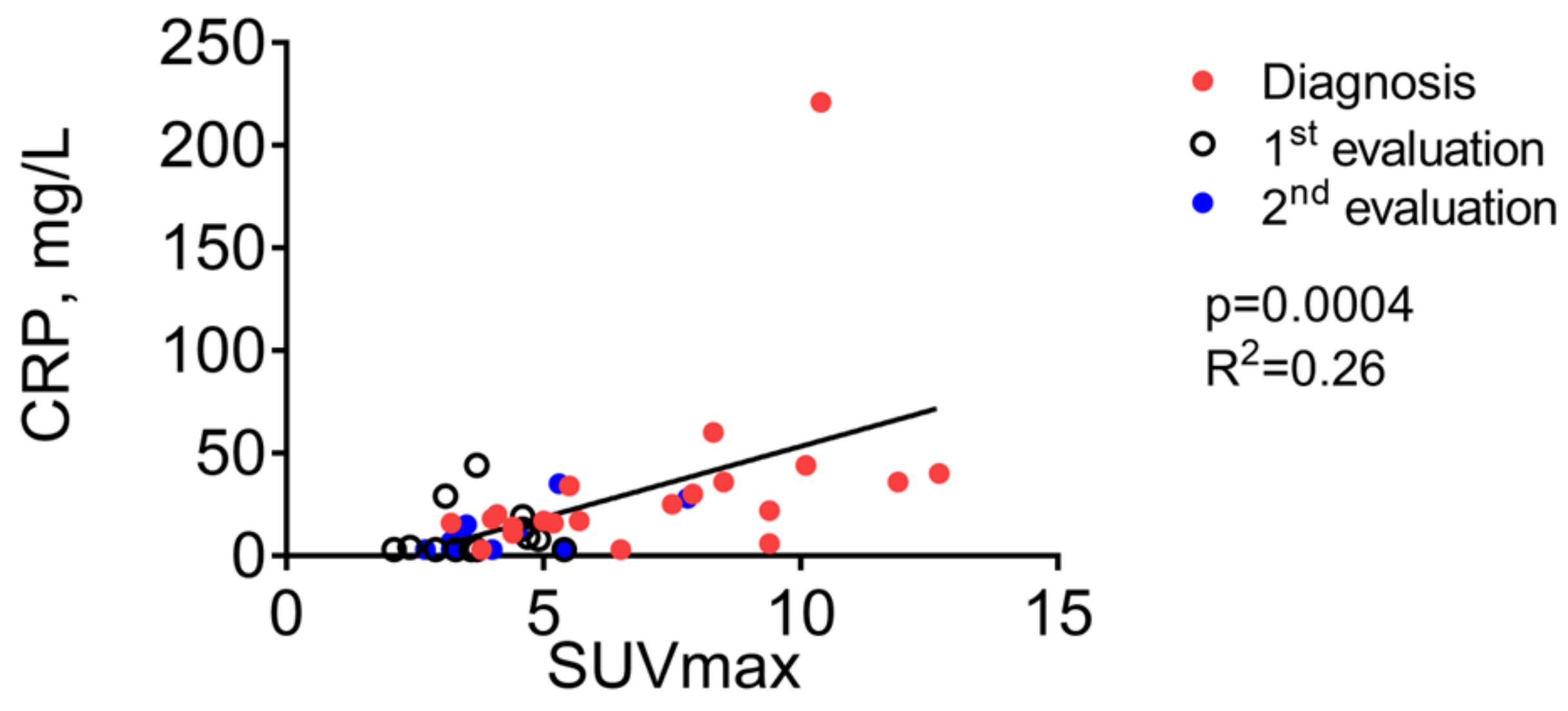




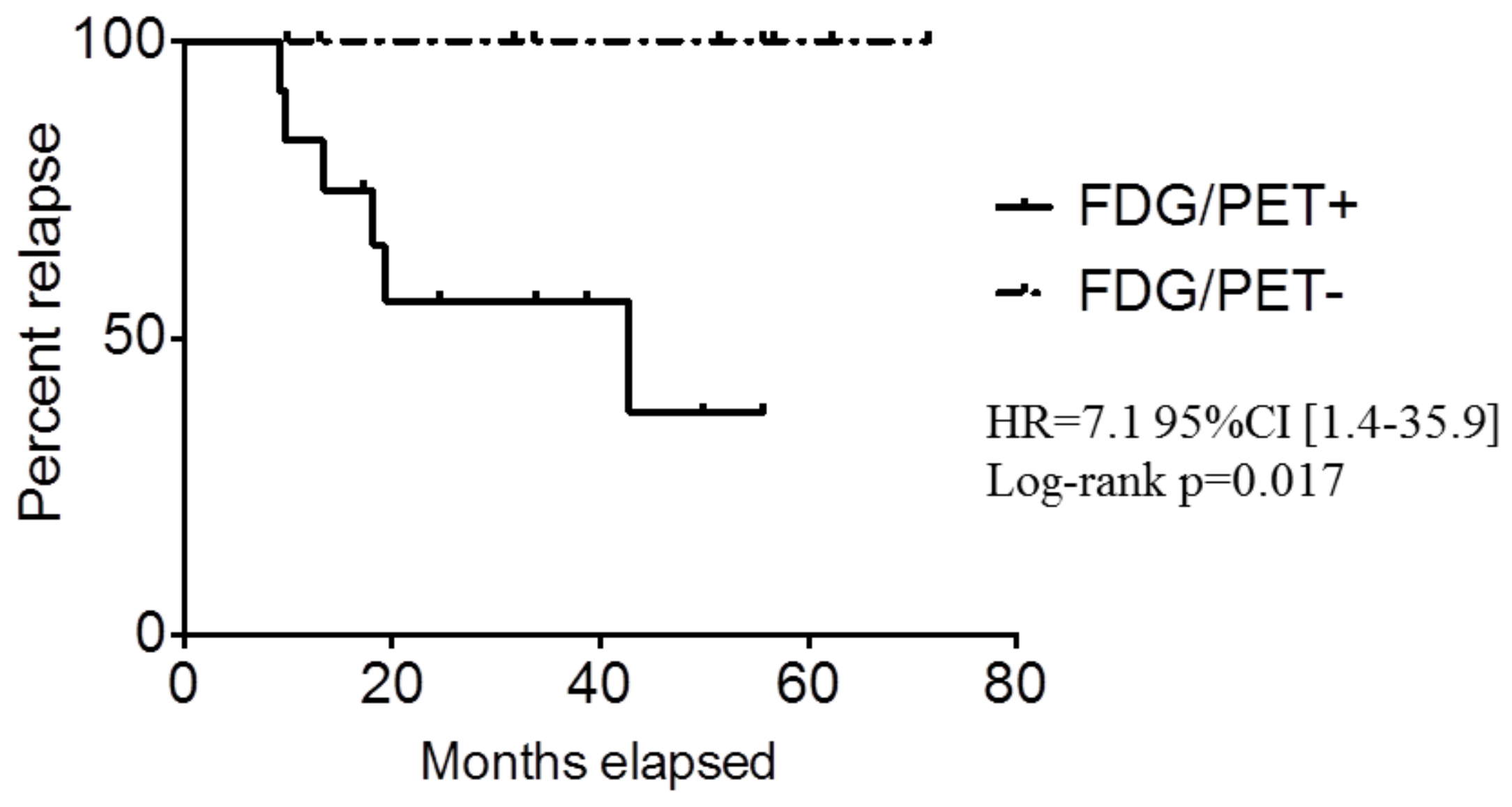

\begin{tabular}{l|rrrrrr} 
Group & 0 & 10 & 20 & 30 & 40 & 50 \\
\hline PET+ & 12 & 11 & 7 & 6 & 4 & 2 \\
PET- & 9 & 9 & 8 & 8 & 6 & 5
\end{tabular}

